\title{
La formación en alfabetización en información en las aulas universitarias: el caso de la UC3M
}

Information literacy in university classrooms: the case of the Carlos III University of Madrid

\author{
Tony Hernández-PÉrez, Ana R. Pacios, Marina Vianello, Raúl Aguilera Ortega (1) \\ y Mayte RAMOS GOROSPE (2)
}

(1) Departamento de Biblioteconomía y Documentación, Universidad Carlos III de Madrid, C/ Madrid 126, 28903 Getafe, España

(2) Universidad Carlos III de Madrid, Avda. Universidad Carlos III 22, 28270 Colmenarejo (Madrid), España

tony@bib.uc3m.es, areyes@bib.uc3m.es, mvianell@bib.uc3m.es, raguiler@db.uc3m.es, mayte@db.uc3m.es

\begin{abstract}
Resumen
La asignatura de "Técnicas de búsqueda y uso de la información" forma parte del curriculum académico de todos los estudiantes que cursan un Grado en la Universidad Carlos III de Madrid desde que se pusieron en marcha las titulaciones adaptadas al Espacio Europeo de Educación Superior. Se trata de una asignatura que tiene por objetivo potenciar la alfabetización informacional de los estudiantes y la adquisición de las competencias básicas como buscar, seleccionar, evaluar, usar y comunicar información. La docencia de esta asignatura la lleva a cabo el Departamento de Biblioteconomía y Documentación con la colaboración del Servicio de Biblioteca que se ocupa de las sesiones prácticas. Después de tres cursos académicos se presenta un balance con la evolución de la asignatura, los resultados académicos y las repercusiones en el uso de los servicios y recursos de la biblioteca.
\end{abstract}

Palabras clave: Alfabetización informacional. Competencias informacionales. Desarrollo curricular. Enseñanza de pregrado. Asignaturas transversales. Colaboración entre bibliotecas y departamentos de biblioteconomía y documentación. Universidad Carlos III de Madrid (España).

\section{Introducción}

Todas las políticas educativas de organizaciones nacionales e internacionales de los últimos años insisten en la necesidad del aprendizaje permanente y en la de modificación del modelo docente predominante hasta ahora. Tal y como se dice en el documento Estrategia Universidad 2015 (España. Ministerio de Educación, 2011):

La cultura docente del profesorado y la cultura del estudiante como aprendiz deben cambiar y deben hacerlo en aras de un modelo de docencia centrado en promover la actividad del estudiante, y de un modelo de aprendizaje que integre esfuerzo, aprendizaje de contenidos con valor estratégico, competencias para el ejercicio profesional y desarrollo de actitudes proactivas en relación al mundo del saber -aprender a aprender-y con el de la iniciativa y la capacidad de emprender".

\begin{abstract}
The course "Information skills" (Searching and using information) is part of the curriculum of all the graduate students at the University Carlos III of Madrid since the academic year 2008-2009. This was the first year when the university adapted all grades to the European Higher Education Area (EHEA). The aim of the course is to promote information literacy among the students and to provide them with the basic skills for searching, selecting, evaluating, using and communicating information in a digital environment. The teaching of the course is carried out by the Library and Information Science Departament with the close collaboration of the Library Service of the University. The library is in charge of several of the practical sessions. After three years, we present a balance with the evolution of the course, academics results and its impact on the use of services and library resources.
\end{abstract}

Keywords: Information literacy. Information skills. Curriculum development. Pregraduate studies. Transversal courses. Collaboration between academic departments and libraries. Carlos III University of Madrid.

Una de las competencias reconocidas por prácticamente todos los organismos internacionales, incluyendo la UE (Verniers, 2009), OCDE (OCDE, 2005), Unesco (UNESCO, 2011), IFLA (Lau, 2006) y demás instituciones educativas nacionales es la competencia en alfabetización de información, entendida como el conjunto de habilidades que necesita un individuo para reconocer cuando tiene una necesidad de información y tiene la habilidad para localizarla, evaIuarla y utilizarla de forma efectiva para satisfacer dicha necesidad (ALA, 2006). Y Pinto y otros hacen un buen repaso conceptual y terminológico del tema (Pinto et al., 2010).

Diversos estudios sobre las competencias y el comportamiento de los alumnos universitarios respecto a las búsquedas de información (Connaway y Dickey, 2010) demuestran que prácticamente el $90 \%$ de los estudiantes comienza a 
buscar en un motor de búsqueda y no en los caros recursos de información en los que invierten las distintas bibliotecas (CIBER, 2008), tanto entre aquellos que están llegando a la universidad en los últimos años, los de la llamada "generación google", como de generaciones algo anteriores. La gran mayoría de estos usuarios se sienten además satisfechos con los resultados que encuentran (OCLC, 2005 y 2010).

Conscientes de la importancia de estas competencias, numerosas universidades de todos los continentes han puesto en marcha programas de formación en alfabetización de información que básicamente se implementan de tres formas: como cursos específicos, generalmente implican la concesión de los créditos correspondientes; como actividades integradas en otros cursos más generales; o como cursos extracurriculares que se imparten fuera de las aulas, a menudo en las bibliotecas, y que generalmente no otorgan ningún crédito al estudiante.

La adaptación al Espacio Europeo de Educación Superior (en adelante, EEES) brindó una oportunidad para integrar la alfabetización en información en el diseño de los planes de estudio. Así lo hizo la Universidad Carlos III de Madrid al crear en el curso 2008-2009 una nueva asignatura transversal obligatoria para todos los estudiantes de primer curso independientemente de la titulación en la que estén matriculados: Técnicas de búsqueda y uso de la información. El Departamento de Biblioteconomía y Documentación imparte la docencia de esta asignatura con la colaboración del Servicio de Biblioteca que se encarga de varias sesiones prácticas. Esta colaboración permite que la experiencia de profesores y bibliotecarios se complemente para ofrecer a los estudiantes una aproximación a la materia desde la perspectiva académica y profesional. Tras la experiencia de tres cursos académicos, se expone la evolución de la asignatura y las mejoras introducidas dirigidas a facilitar el aprendizaje, disminuir las diferencias en cuanto a los contenidos comunes que se deben impartir y la tipología de prácticas a realizar en todos los grados, así como a mejorar el sistema de evaluación adaptándolo a la normativa de la universidad.

\section{El marco de la alfabetización en información en la Universidad Carlos III de Madrid}

\subsection{1. La reforma de los planes de grado}

En 2007 la Universidad Carlos III de Madrid se puso a trabajar en el diseño de los nuevos planes de estudio adaptados al EEES, implantados en el curso 2008-2009 tras su verificación por el Consejo de Universidades. Siguiendo las directrices del Real Decreto 55/2005, de 21 de enero, por el que se regulan los estudios universitarios de Grado, para obtener el título de Graduado el estudiante debe obtener 240 créditos ECTS, que en la Universidad Carlos III se articulan en titulaciones de cuatro cursos. Las asignaturas de que constan estos planes de estudio son de diversos tipos: obligatorias, optativas, formación básica, prácticas y trabajo fin de grado. Dentro de esta tipología, las asignaturas consideradas "formación básica" deben tener un mínimo de 60 créditos, pero si bien al menos 36 de ellos deben estar vinculados a la rama de conocimiento de la titulación, los créditos restantes dejan un amplio margen de maniobra.

En este sentido, la Universidad Carlos III de Madrid decidió utilizar 18 créditos para poner en marcha una formación transversal común a todas las titulaciones y distribuida, además, entre los dos primeros cursos. Desde su fundación en 1989, la Universidad Carlos III de Madrid ha apostado por un modelo de universidad pública comprometido con la calidad de la docencia, la excelencia en la investigación y la formación de ciudadanos críticos y maduros. Se aspira a que los egresados sean buenos profesionales, pero también se persigue que sean personas cultas, solidarias con los demás y sensibles hacia el medio ambiente. De ahí que se destinaran 6 créditos al inglés, 6 créditos para actividades o cursos de Humanidades y los 6 créditos restantes se dedicaran a las competencias en información y expresión oral y escrita. Estos 6 créditos se estructuran en dos asignaturas diferentes, cada una de ellas de 3 créditos ECTS: Técnicas de expresión oral y escrita y Técnicas de búsqueda y uso de la información.

De esta manera la Universidad satisfacía las demandas de un colectivo esencial como el de los estudiantes, quienes entre sus propuestas para los nuevos planes de Grado defendían la existencia de alguna asignatura sobre gestión de la información. Además, al basarse los contenidos de esta asignatura en la alfabetización en información se pusieron en práctica las recomendaciones de los libros blancos de los títulos de Grado elaborados por la Agencia Nacional de Evaluación de la Calidad y Acreditación (ANECA) y de la Red de Bibliotecas Universitarias (en adelante, REBIUN) en su Documento Marco (Area, 2007) sobre adquisición de este tipo de competencias. La asignatura Técnicas de búsqueda y uso de la información en la Universidad Carlos III de Madrid nació así con el impulso de las autoridades académicas, la conformidad de los estudiantes y el acuerdo del 
Servicio de Biblioteca y del Departamento de Biblioteconomía y Documentación para colaborar en el proceso de enseñanza-aprendizaje de la asignatura.

A diferencia de otras universidades, en las que las competencias informacionales se insertan como parte de otras asignaturas - por ejemplo en el Grado en Enfermería en la Universidad de Sevilla o en la Universidad de Granada- o en cursos voluntarios, con o sin reconocimiento de créditos -véase el buen ejemplo del proyecto colaborativo en $\mathrm{Cl} 2$ (Competencias informacionales e informáticas) de las universidades de La Laguna, Santiago, Zaragoza y Alicante-, la Carlos III decidió apostar por una asignatura específica y en los primeros cursos de grado y no en los cursos finales, como puede ser el caso de otras universidades en donde está información se dirige a alumnos de últimos cursos de carrera o posgrados, como es el caso de la Universidad Politécnica de Catalunya en la cual estos cursos forman también parte de la docencia reglada. Como se observa, no existe un modelo único ni se puede afirmar que un modelo sea mejor que otro pues las circunstancias de cada universidad son muy diferentes. REBIUN dedicó en 2009 unas jornadas al tema y los materiales de la plataforma ALFARED permiten conocer la situación actual de lo que se está haciendo en las bibliotecas universitarias españolas, así como las mejores prácticas.

\subsection{Nueva metodología,} nuevas infraestructuras

El diseño de los nuevos planes de estudio es un requisito previo para la adaptación al EEES, pero el esfuerzo no se puede agotar aquí. Para que el EEES sea una realidad debe cambiar la metodología docente y de aprendizaje y, por lo tanto, debe implicar asimismo una renovación de las infraestructuras universitarias (Area, 2007 y Area, Gros y Marzal, 2008).

La Universidad Carlos III de Madrid viene trabajando en estos dos aspectos de la adaptación al EEES. Desde el punto de vista metodológico, el trabajo del alumno, ya sea personal o colectivo, en clase o en casa, cuenta como mínimo un $40 \%$ de la calificación final. La última normativa sobre evaluación continua, aprobada el 31 de Mayo del 2011, pone de manifiesto la necesidad de ir modificando los procedimientos conforme a las sugerencias y propuestas de los profesores en función de la experiencia adquirida en estos años.

El sistema de evaluación continua obliga al profesor a reconocer el esfuerzo y la implicación activa del estudiante. Para reforzar la dimensión práctica de las clases, los grupos están formados por un máximo de 40 alumnos, de ahí que las antiguas aulas de docencia se hayan venido reformando poco a poco desde que empezaron a extinguirse los cursos de los planes antiguos. Una herramienta clave en el desarrollo de esta metodología es la plataforma de aprendizaje a distancia, denominada Aula Global 2 y que utiliza el difundido software Moodle. Alumnos y profesores del curso de Técnicas de búsqueda y uso de la información disponen en Aula Global 2 de los materiales didácticos y de las herramientas de apoyo a la docencia. Sirve también como sistema de comunicación entre profesores y bibliotecarios, involucrados en la docencia de la asignatura. Ambos comparten un espacio común en el que los bibliotecarios ponen a disposición de los profesores los materiales que sirven de guía para las visitas y las clases sobre los recursos de información que enseñan a utilizar a los alumnos, así como ejercicios prácticos.

En paralelo se ha acometido la reforma de las aulas informáticas para que todas cuenten con cañón proyector y pantalla y sean aptas para la impartición de clases. Evidentemente, la asignatura de Técnicas de búsqueda y uso de la información sigue estos mismos criterios de evaluación continua y da especial importancia al uso de aulas informáticas para el acceso a las fuentes de información y el uso de diversas herramientas de gestión de la información.

\subsection{El Servicio de Biblioteca}

Entre las infraestructuras universitarias involucradas en la implantación del EEES y la transformación del modelo de aprendizaje tiene especial relieve el Servicio de Biblioteca. El Servicio de Biblioteca de la Universidad Carlos III ha trabajado en los últimos años para ser un verdadero Centro de Recursos para el Aprendizaje y la Investigación (en adelante, CRAI), en línea con el modelo de biblioteca universitaria impulsado desde la Red de Bibliotecas Universitarias (en adelante, REBIUN) en su I Plan Estratégico 2003-2006 y su II Plan Estratégico 2007-2010.

Las bibliotecas de la Universidad Carlos III ofrecen diversos espacios y servicios para el aprendizaje. Entre los espacios, cabe resaltar la creación de salas de trabajo en grupo, la mejora de las salas con equipamiento informático para digitalización o las aulas de idiomas. Y entre los servicios, quizá el más relevante para la adaptación al EEES es la formación de usuarios.

La formación de usuarios comenzó como tal servicio desde el momento en que nació el Servicio de Biblioteca con la Universidad en 1989. Es, sin embargo, en 1992, cuando se empeza- 
ron a programar sesiones formativas en las propias instalaciones de las bibliotecas con plena participación de todo el personal bibliotecario. En 1993 se consolidó la formación básica a los alumnos de nuevo ingreso durante las primeras semanas de curso, así como también se empezaron a dar los primeros cursos especializados en algunas titulaciones; en 1998 se inició la oferta de cursos programados, personalizados y a la carta sobre el uso de recursos de información de todo tipo; y en 2001 comenzó la oferta de cursos a profesores e investigadores.

El Plan Estratégico 2005-2007 incluyó la formación como una línea estratégica del servicio, sobre todo ante la inminente implantación del EEES, y promovió la elaboración de un primer plan de formación que, entre otros objetivos, apostaba por la integración de un programa de alfabetización en información dirigido a toda la comunidad universitaria.

Ya en el contexto del Plan Estratégico 20082011, que profundiza en esta línea y ha permitido realizar significativas mejoras organizativas, surgió la posibilidad de colaborar con el Departamento de Biblioteconomía y Documentación en algunas de las sesiones prácticas de la nueva asignatura de Grado sobre Técnicas de búsqueda y uso de la información. Desde el curso 2008-2009 el Servicio de Biblioteca contribuye así con su experiencia en la gestión de información y el uso de fuentes de información, sin que esto signifique haber abandonado los cursos de formación, especialmente los dirigidos a PDI.

\section{La asignatura de técnicas de búsqueda y uso de la información}

\subsection{Funcionamiento}

La asignatura de Técnicas de búsqueda y uso de la información se encuadra transversalmente dentro de las asignaturas de formación básica de todas las titulaciones de Grado. Excepto en una titulación, se imparte en primer curso; en algunos casos se encuentra en el primer cuatrimestre y en otros, en el segundo. Esta asignatura tiene 3 créditos ECTS y, en función del calendario académico de los diferentes grupos, se alterna dentro de un mismo cuatrimestre con la asignatura de Técnicas de expresión oral y escrita. De los 3 créditos ECTS, tres horas semanales son de asistencia y trabajo en clase, y 7 horas son de trabajo personal del alumno. En total la asignatura se imparte durante 7 semanas, lo que hace un total de 14 sesiones El número de grupos totales por curso académico ha variado en los tres años, pasando de 104 a 126.
Otro detalle importante de la asignatura de Técnicas de búsqueda y uso de la información es su carácter práctico. Si bien en otras asignaturas varios grupos de 40 alumnos como máximo pueden juntarse periódicamente en clases magistrales, en ésta los conocimientos teóricos van de la mano de su aplicación práctica en cada una de las dos sesiones semanales de hora y media que tiene. Inicialmente los alumnos contaron con una sesión en aula convencional y otra en aula informática.

La demanda, tanto por parte de profesores como de alumnos, de un mayor número de prácticas ha llevado a organizar la docencia de las dos clases semanales en aula informática a partir del curso 2011-2012 en las titulaciones donde ha sido posible.

Los alumnos disponen en Aula Global 2 de los materiales didácticos y de herramientas de comunicación con sus profesores y compañeros. Para facilitar el seguimiento de la asignatura se ofrece al alumno un cronograma detallado (Figura 1).

Uno de los principales retos de la puesta en marcha de la asignatura en estos años ha sido impartir su docencia en inglés en algunos grupos de titulaciones bilingües (grados en Economía, Administración de Empresas, Periodismo, Comunicación Audiovisual, Finanzas y Contabilidad, así como las ingenierías). Los profesores y los bibliotecarios están haciendo un gran esfuerzo con estos grupos, veinticinco en el curso 2010-2011, que representan la quinta parte del total de grupos de la asignatura.

\subsection{Contenidos: alfabetización en información}

El programa de la asignatura de Técnicas de búsqueda y uso de la información es común en objetivos, contenidos y estructura a todas las titulaciones, aunque lógicamente los recursos de información especializados cuyo uso se enseña varían de una titulación a otra. El objetivo de la asignatura es facilitar la adquisición de las competencias básicas en información por los estudiantes, con las miras puestas no sólo en sus estudios universitarios sino también en su futuro ejercicio profesional. La finalidad es lograr que las habilidades adquiridas por los alumnos les permitan desarrollar su aprendizaje de forma autónoma y a lo largo de toda su vida, que es algo que está en el centro de la concepción educativa del Espacio Europeo de Educación Superior. 


\begin{tabular}{|c|c|c|c|c|c|c|}
\hline \multirow{2}{*}{ 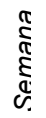 } & \multirow{2}{*}{ :̊ } & \multirow[t]{2}{*}{ Descripción del contenido de la sesión } & \multirow{2}{*}{$\begin{array}{l}\text { Espacio } \\
\text { necesario }\end{array}$} & \multicolumn{3}{|c|}{ Horas de trabajo del alumno durante la semana } \\
\hline & & & & Descripción & Presencial & Personal \\
\hline 1 & 1 & $\begin{array}{l}\text { Presentación de la asignatura } \\
\text { Explicación de las trabajos prácticos y del trabajo final } \\
\text { en grupo. } \\
\text { Tema 1: Dónde encontrar información fiable } \\
\text { - Datos, información y conocimiento } \\
\text { - Competencias en información } \\
\text { Presentación de material informativo de los servicios } \\
\text { de la biblioteca }\end{array}$ & $\begin{array}{l}\text { Aula } \\
\text { informática }\end{array}$ & $\begin{array}{l}\text { Formación de grupos para } \\
\text { el trabajo final } \\
\text { Lecturas complementarias } \\
\text { al tema }\end{array}$ & 1,5 & 7 \\
\hline 1 & 2 & $\begin{array}{l}\text { Tema 1: Dónde encontrar información fiable } \\
\text { · Fuentes de información: tipología y localización }\end{array}$ & $\begin{array}{l}\text { Aula } \\
\text { informática }\end{array}$ & $\begin{array}{l}\text { Realización de ejercicios } \\
\text { prácticos. } \\
\text { Realización de } \\
\text { cuestionario de autoeva- } \\
\text { luación del tema } 1 . \\
\text { Inicio del trabajo en Grupo } \\
\text { Realización del cuestiona- } \\
\text { rio de autoevaluación } \\
\text { Biblioteca }\end{array}$ & 1,5 & \\
\hline 2 & 3 & $\begin{array}{l}\text { Tema 1: Dónde encontrar información fiable } \\
\text { - Evaluación de los recursos informativos }\end{array}$ & $\begin{array}{l}\text { Aula } \\
\text { informática }\end{array}$ & $\begin{array}{l}\text { Realización de ejercicios } \\
\text { prácticos. } \\
\text { Lecturas complementarias } \\
\text { al tema }\end{array}$ & 1,5 & 7 \\
\hline 2 & 4 & $\begin{array}{l}\text { Tema 2: Uso ético de la información: Citación y } \\
\text { Bibliografía } \\
\text { - Ética y propiedad intelectual } \\
\text { - El trabajo académico sin plagio } \\
\text { - Crear citas y referencias bibliográficas }\end{array}$ & $\begin{array}{l}\text { Aula } \\
\text { informática }\end{array}$ & $\begin{array}{l}\text { Realización de ejercicios } \\
\text { prácticos (trabajo en } \\
\text { grupo). } \\
\text { Lecturas complementarias } \\
\text { al tema }\end{array}$ & 1,5 & \\
\hline
\end{tabular}

Figura 1. Cronograma de la asignatura de Técnicas de búsqueda y uso de la información en el Grado en Administración de Empresas (curso 2011-2012)

Asimismo, un rápido vistazo a la asignatura de Técnicas de búsqueda y uso de la información pone de manifiesto que sus contenidos se corresponden con la alfabetización en información. En efecto, en esta asignatura se enseñan competencias en la búsqueda, selección, evaluación, uso y comunicación de la información, que son las cinco competencias básicas en que coinciden, con mayor o menor precisión, organizaciones internacionales como la International Federation of Library Associations (IFLA) (Lau, 2006), la Association of College and Research Libraries de Estados Unidos (ACRL, 2000), la Society of College, National and University Libraries del Reino Unido (SCONUL, 1999) o el Council of Australian and New Zealand Institute for Information Literacy (ANZIIL) de Australia y Nueva Zelanda (Bundy, 2004), por poner sólo los ejemplos más conocidos.

En el diseño del programa se tuvieron muy en cuenta las ideas de Shapiro y Hughes (1996) con el fin de incluir habilidades relacionadas con los recursos generales y especializados, para comprender la forma, el formato, la localización y los métodos de acceso a los recursos de información; con la investigación, para comprender cómo se realizan los procesos de descubri- miento, investigación y uso de los recursos; con la publicación, para iniciar a los alumnos en los procesos de producción de informes, citación y en la ética de la información; y con las herramientas, para que sean capaces de utilizar de forma eficiente los motores de búsqueda pero también otros recursos en formato digital.

La asignatura tiene los siguientes temas con sus respectivos contenidos, como se puede ver en el programa común para todos los Grados del curso 2011-12:

1. Dónde encontrar información fiable

—Datos, información y conocimiento

- Competencias en información

-Fuentes de información: tipología y localización

—Evaluación de los recursos informativos

2. Uso ético de la información: citación y bibliografía

$$
\begin{aligned}
& \text {-Ética y propiedad intelectual } \\
& \text {-El trabajo académico sin plagio } \\
& \text { - Crear citas y referencias bibliográficas }
\end{aligned}
$$


- Cómo presentar y ordenar las referencias bibliográficas

-Programas informáticos para la gestión de citas y bibliografía

3. Recuperación de la información en entornos electrónicos: recursos generales

\section{- Conceptos básicos}

-Procesos en la búsqueda de información electrónica

- Bases de datos multidisciplinares

- Herramientas de búsqueda en Internet

4. Recuperación de la información en entornos electrónicos: recursos especializados

- Portales y bases de datos especializadas

- Selección de recursos en la Red

- Nuevos espacios de interacción con el conocimiento

A medida que se ha venido impartiendo la asignatura se ha puesto mayor énfasis en la importancia de hacer un uso ético de la información. Resulta esencial concienciar a los alumnos contra el plagio explicándoles que el uso de la información no es libre y puede estar sujeto a condicionantes éticos y legales, así como enseñándoles a seleccionar y evaluar los recursos de información y a usar correctamente el sistema de citas de la comunicación científica. En este sentido la Universidad Carlos III de Madrid ha adquirido Turnitin, una herramienta que permite verificar la originalidad de los trabajos haciendo evidente los peligros del plagio y ayudando así a los estudiantes y a los profesores a identificarlos y corregir los problemas.

\subsection{Coordinación y organización}

La responsabilidad de la organización de la asignatura recae en la figura de una de las subdirecciones del Departamento que se encarga de la asignación de la docencia, de realizar el seguimiento y analizar cualquier problema que surja en su impartición. Asimismo son temas de su responsabilidad las actuaciones dirigidas a consensuar eventuales cambios en el temario de la asignatura.

El hecho de que la asignatura se imparta en todos los grados que ofrece la universidad y que, a menudo, haya más de un grupo para cada grado, hace necesario contar con un coordinador para cada uno de ellos. Sus tareas consisten en: orientar a los profesores que se incorporan a su docencia, velar para que en todos los grupos se asigne la misma carga de trabajo y se apliquen los mismos criterios de evaluación, y estar en contacto con la biblioteca para acordar los contenidos de sus sesiones. A ello hay que añadir la selección de fuentes y la adaptación de ejercicios a la materia de cada grado.

Finalmente, es el coordinador el que participa en las Comisiones Académicas de la Titulación tratando de sensibilizar al resto del profesorado para que exijan en sus asignaturas la aplicación de las técnicas aprendidas por los estudiantes en esta asignatura.

\subsubsection{La colaboración entre el Departamento y la Biblioteca}

Desde su diseño, la asignatura de Técnicas de búsqueda y uso de la información se ha interpretado como un proyecto de colaboración entre el Departamento de Biblioteconomía y Documentación y el Servicio de la Biblioteca, conscientes de que de este encuentro la docencia se beneficiaría de la experiencia de los bibliotecarios en el uso de las fuentes de información especializadas y el personal de la biblioteca tendría la oportunidad de entrar en contacto con un importante número de alumnos. Es importante resaltar que desde el inicio, tanto por parte del personal docente del Departamento -que está implicado casi en su totalidad en la impartición de esta asignatura-, así como de los bibliotecarios, la implicación ha sido generosa y la colaboración, sin recelos.

Ante el esfuerzo que iba suponer asegurar la docencia de una asignatura que se imparte en todos los nuevos grados implantados en la Universidad se analizaron diversas fórmulas de colaboración. Finalmente el modelo elegido consistió en integrar en el programa de la asignatura la participación de la Biblioteca, que en la actualidad se encarga de la organización de una visita guiada a la biblioteca para todos los alumnos de primer curso y de dos sesiones presenciales, además de proporcionar materiales formativos e instrumentos de evaluación. Todas las actividades realizadas por la biblioteca son parte obligatoria de los contenidos por los que se evalúa al estudiante. Esto ha supuesto un gran trabajo de coordinación y ha obligado a establecer un modelo organizativo capaz poner en marcha el proyecto (Figura 2).

Con este fin, desde la fase de preparación del curso, la coordinación académica de la asignatura, responsabilidad del Departamento de Biblioteconomía y Documentación, empezó a trabajar estrechamente con la coordinación de la línea estratégica de adaptación al EEES de las infraestructuras de aprendizaje del Servicio de Biblioteca. Para facilitar la colaboración y 
compartir materiales, el Departamento habilitó un espacio en una instalación de Moodle de su servidor. La Biblioteca dispone de una carpeta donde se pone a disposición de los profesores toda la documentación relativa a su participación en la asignatura, desde los documentos de planificación hasta las presentaciones que utilizan en las clases.

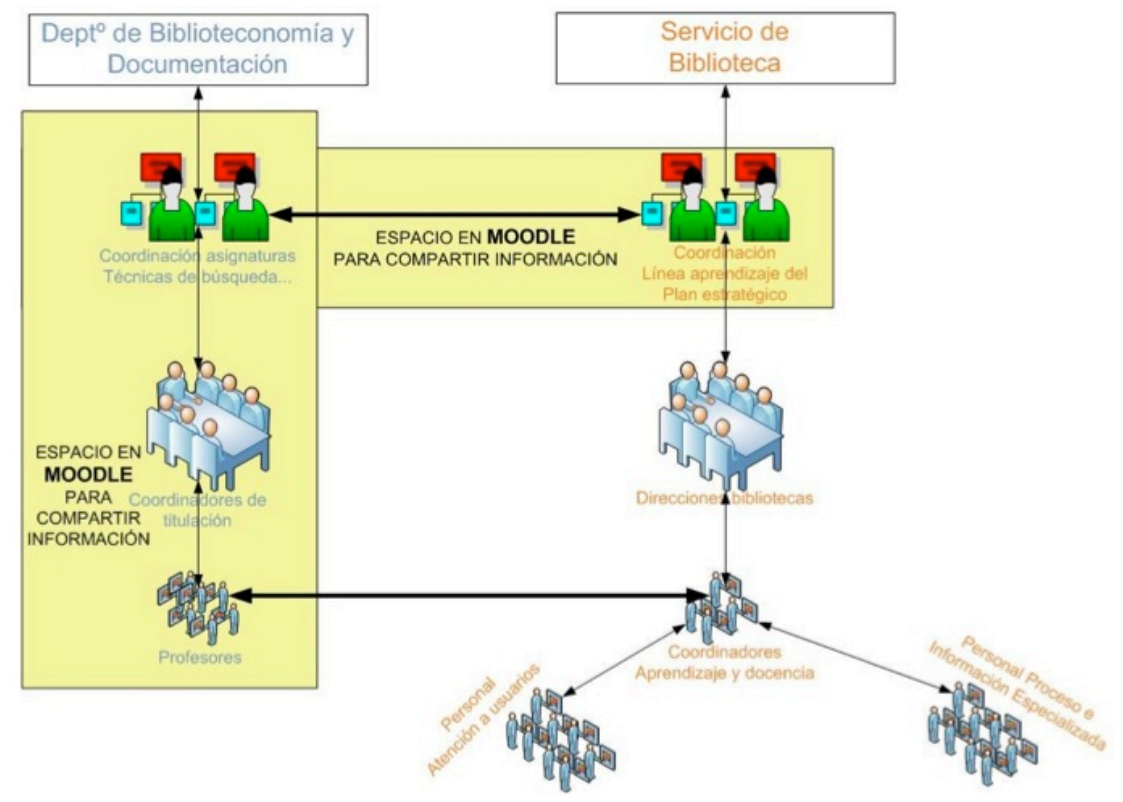

Figura 2. Mecanismos de coordinación entre el departamento y el servicio de biblioteca

Esta iniciativa ha resultado fructífera y en la actualidad, más allá del uso organizativo, es habitual el intercambio de materiales y sugerencias entre profesores y bibliotecarios, habiéndose utilizando en algunos casos también el foro para tomar el pulso a la andadura de la asignatura y diseñar de forma más eficaz las reuniones que periódicamente se organizan para poner en común experiencias y aclarar dudas. Desde el curso 2009-10 la herramienta utilizada para compartir información y materiales es Aula Global 2.

Del mismo modo que la coordinación de la asignatura en el Departamento trabajaba directamente con los profesores que desarrollaban funciones de coordinación dentro de cada titulación y éstos, a su vez, con los profesores de los distintos grupos a su cargo, en la Biblioteca los coordinadores de la línea estratégica de aprendizaje trabajaban con las direcciones de los centros y los responsables de las secciones de Atención a Usuarios y Proceso e Información Especializada; y éstos, a su vez, con el resto de bibliotecarios.

En 2009, para impulsar las necesidades organizativas de todas las tareas de formación y las relacionadas con la Biblioteca 2.0 y el Taller del Aula, unidad encargada del apoyo al profesorado en Aula Global, se creó la figura de la Coor- dinación de Aprendizaje y Docencia en las tres bibliotecas más grandes. Este esquema de colaboración permite desarrollar con garantías toda la actividad de la asignatura.

En definitiva, en los tres cursos académicos en los que se ha venido impartiendo la asignatura 50 bibliotecarios de los tres campus (27 bibliotecarios de atención a usuarios en las visitas guiadas y 23 bibliotecarios temáticos en las sesiones prácticas de acceso a los recursos electrónicos) han tenido la oportunidad cada año de tener contacto directo con 3.000 alumnos de 120 grupos a lo largo de 180 visitas guiadas y 240 sesiones prácticas (2 sesiones cada grupo), lo que constituye un total de 900 horas anuales de trabajo.

\subsubsection{Contenidos de las sesiones en las que participa la Biblioteca}

En el curso 2008-2009 se decidió que la Biblioteca participara en 3 de las 14 sesiones de la asignatura, de hora y media cada una. De las tres sesiones, la primera se orientó hacia la formación básica de los alumnos en el uso de los servicios bibliotecarios, el conocimiento general de las colecciones bibliográficas impresas y electrónicas, y el manejo de las herramientas de búsqueda de información y servicios electrónicos del catálogo automatizado. 
Las otras dos sesiones, en cambio, se reservaron para la explicación de algunas de las bases de datos especializadas más importantes en las distintas áreas de conocimiento científico. Algunas bases de datos son comunes a todos los grados y otras son para grados específicos (Aguilera et al, 2010). Por ello las clases se desarrollan siempre en aula informática. Estas sesiones consisten en la explicación de las principales funcionalidades de las bases de datos acompañadas de búsquedas de información. Diversas presentaciones sirven como material de apoyo y, además de los ejercicios realizados en clase, se proponen al profesor algunas prácticas que le permitan evaluar a los alumnos.

A partir del curso 2009-2010 se suprimió la primera sesión de carácter presencial y se ha sustituido por un material formativo que se ha ampliado y mejorado y que se titula Aprende a usar las bibliotecas. Ahora estos contenidos y actividades entran plenamente dentro de las horas de trabajo personal del alumno. Además la Biblioteca organiza visitas guiadas a los centros.

Sí se mantienen las dos sesiones de formación especializada, pero también aquí se han introducido significativas mejoras en los contenidos de las sesiones y los materiales formativos en todos los cursos académicos. Se han orientado las prácticas de clase y los ejercicios que se proponen hacia la resolución de problemas, profundizando en lo que se empezó a hacer en algunas titulaciones en el curso 2008-2009.

\section{Los resultados académicos y la satisfacción de los alumnos (2008-2011)}

Para comprender la magnitud del proyecto es necesario conocer el número de alumnos matriculados en la asignatura cada año y apreciar los resultados académicos de la asignatura de Técnicas de búsqueda y uso de la información, que han sido muy positivos, como se ha transmitido oficialmente desde el Vicerrectorado de Grado al Departamento de Biblioteconomía y Documentación y al Servicio de Biblioteca. En la Tabla I se muestran los datos de matrícula y los porcentajes de superación de la asignatura, en torno al $88 \%$, de los alumnos matriculados.

Un pequeño porcentaje de alumnos ha tenido que repetir la asignatura. Si el alumno la cursa en el primer cuatrimestre y no la supera tiene la posibilidad de matricularse y repetirla en el mismo curso académico. Esta situación anómala se debe a que la universidad, ante los retrasos en la matriculación que origina el examen de la Selectividad en septiembre, ofrece esta opción a los alumnos que se incorporan tarde en algunos Grados y no la han podido cursar en su momento.

\begin{tabular}{lccc}
\hline Estudiantes & $\begin{array}{c}2008- \\
2009\end{array}$ & $\begin{array}{c}2009- \\
2010\end{array}$ & $\begin{array}{c}2010- \\
2011\end{array}$ \\
\hline Matriculados & 3.598 & 3.116 & 2.935 \\
\hline Superan la & 3.149 & 2.741 & 2.610 \\
asignatura & $(87,50 \%)$ & $(87,96 \%)$ & $(88,94 \%)$ \\
\hline
\end{tabular}

Tabla I. Relación entre los alumnos que se matriculan y los que superan la signatura

Las encuestas de evaluación de la docencia que cumplimentan los alumnos al finalizar la asignatura permiten aproximarnos a su grado de satisfacción, teniendo en cuenta que la tasa de participación, aunque varía según las titulaciones, se sitúa en torno al $50 \%$. En relación con la pregunta que persigue obtener una valoración global de la docencia (del 1 al 5), se puede afirmar que los valores medios obtenidos por la asignatura durante los tres cursos académicos han superado los valores medios de la titulación en el $50 \%$ de los Grados.

Por otra parte, los comentarios vertidos por los alumnos muestran la utilidad de la asignatura, destacando el conocimiento y la exploración de los recursos informativos que se ponen a su disposición a través del servicio de biblioteca, así como el manejo profesional de los principales motores de búsqueda de información en Internet. A continuación, en la Figura 3, se muestra la valoración (también del 1 al 5) y su evolución durante los tres cursos académicos de cuatro preguntas, de entre las 11 que forman parte de la encuesta, que se han elegido por considerarse representativas de la planificación de la docencia, el desarrollo de la enseñanza y los resultados de aprendizaje conseguidos en la asignatura.

- Pregunta 1: Los objetivos de la asignatura están claros y las tareas previstas (teoría, práctica, de trabajo individual, en grupo...) guardan relación con dichos objetivos.

- Pregunta 2: Valore la coordinación que se ha producido entre las clases de docencia teórica, docencia aplicada y docencia experimental-laboratorios (en el caso de que existan) previstas en el programa.

- Pregunta 3: Valore la utilización de los recursos didácticos e infraestructuras (audiovisuales, de laboratorio, bibliográficos, de campo...) para facilitar el aprendizaje. 
- Pregunta 4: Estime el nivel de habilidades alcanzado (trabajo en equipo, capacidad de resolución de problemas, capacidad organizativa...).

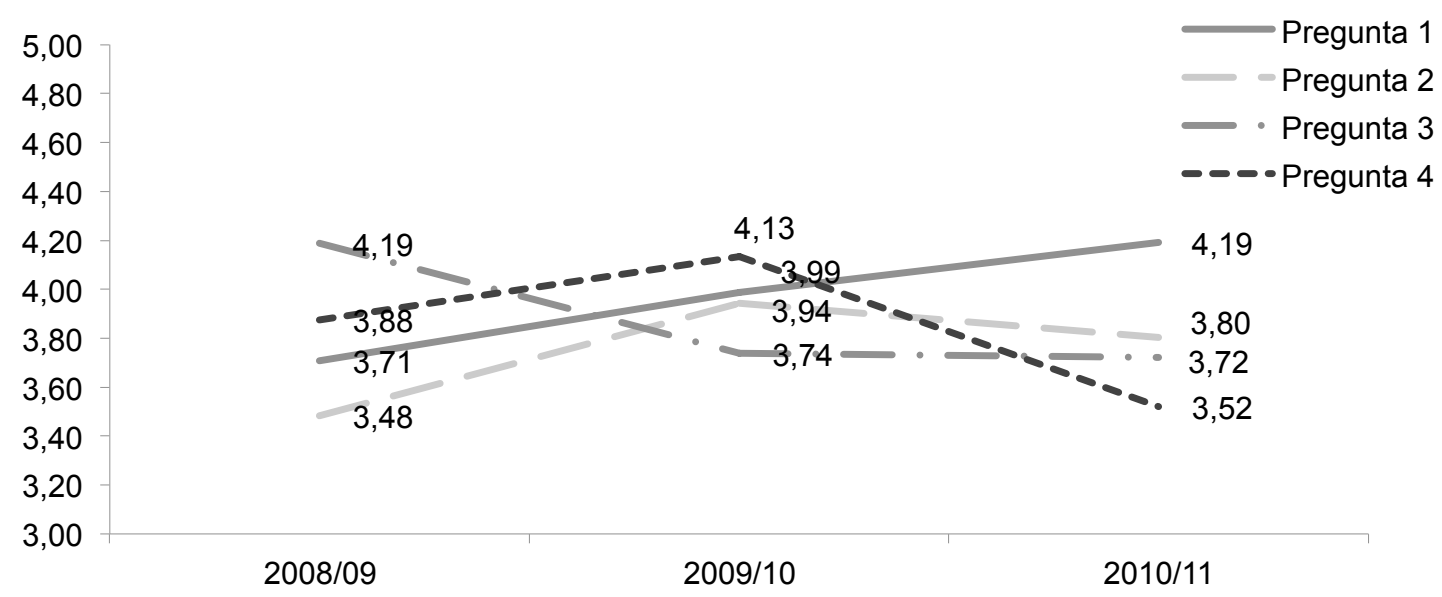

Figura 3. Preguntas y valores medios obtenidos de la asignatura TBUI

Resulta difícil extraer conclusiones con respecto a la evolución durante estos tres años, donde la mejora se advierte en la adecuación entre los objetivos de la asignatura y las tareas realizadas. Las infraestructuras (aulas convencionales para la teoría y aulas informáticas para las prácticas, y los recursos didácticos) se han mantenido en el mismo nivel de calidad, pero han recibido una puntuación decreciente por los estudiantes.

Posiblemente haya que achacarlo a problemas relacionados con el mal funcionamiento en ocasiones de los ordenadores, caídas de la red, algún equipamiento deficiente, etc., a los que han hecho también referencia los alumnos en los comentarios que acompañan a la encuesta. El dato más llamativo es la bajada en la estimación del nivel de habilidades alcanzado, pues el nivel académico de los alumnos, a juzgar por los resultados, ha sido similar todos los años. Por otro lado, también se considera que ha empeorado la coordinación entre las clases teóricas y prácticas previstas en el programa, si bien el modelo de cooperación entre docentes y bibliotecarios está consolidado después de tres años y se viene trabajando anualmente en la mejora de la coordinación y comunicación entre ambos.

\section{Influencia sobre el acceso a los recursos electrónicos}

También sobre los servicios de la Biblioteca ha tenido gran impacto la asignatura de Técnicas de búsqueda y uso de la información, en especial en el acceso a los recursos electrónicos. El cambio de sistema de acceso producido a finales de 2009, que permitió avanzar de un simple listado de recursos a su integración en Universo-e (basado en Metalib) hace que los datos sean difíciles de comparar, sobre todo por perfil de usuario. No obstante, el número total de accesos de todos los perfiles de usuarios puede dar una medida aproximada del notable incremento producido entre los cursos 2007-2008, antes de la puesta en marcha de la asignatura, y el curso 2010-2011 (Tabla II y Figura 4).

\begin{tabular}{lrrr}
\hline Mes & $2007-2008$ & $2010-2011$ & Incremento \\
\hline Septiembre & 5.711 & 17.049 & $199 \%$ \\
\hline Octubre & 21.031 & 37.922 & $80 \%$ \\
\hline Noviembre & 25.248 & 34.694 & $37 \%$ \\
\hline Diciembre & 14.885 & 20.768 & $40 \%$ \\
\hline Enero & 15.354 & 17.542 & $14 \%$ \\
\hline Febrero & 12.046 & 20.149 & $67 \%$ \\
\hline Marzo & 21.232 & 25.394 & $20 \%$ \\
\hline Abril & 29.213 & 18.026 & $-38 \%$ \\
\hline Mayo & 19.074 & 19.540 & $2 \%$ \\
\hline Junio & 11.080 & 51.320 & $363 \%$ \\
\hline Total & 174.874 & 262.404 & $50 \%$ \\
\hline
\end{tabular}

Tabla II. Comparación del número de accesos 


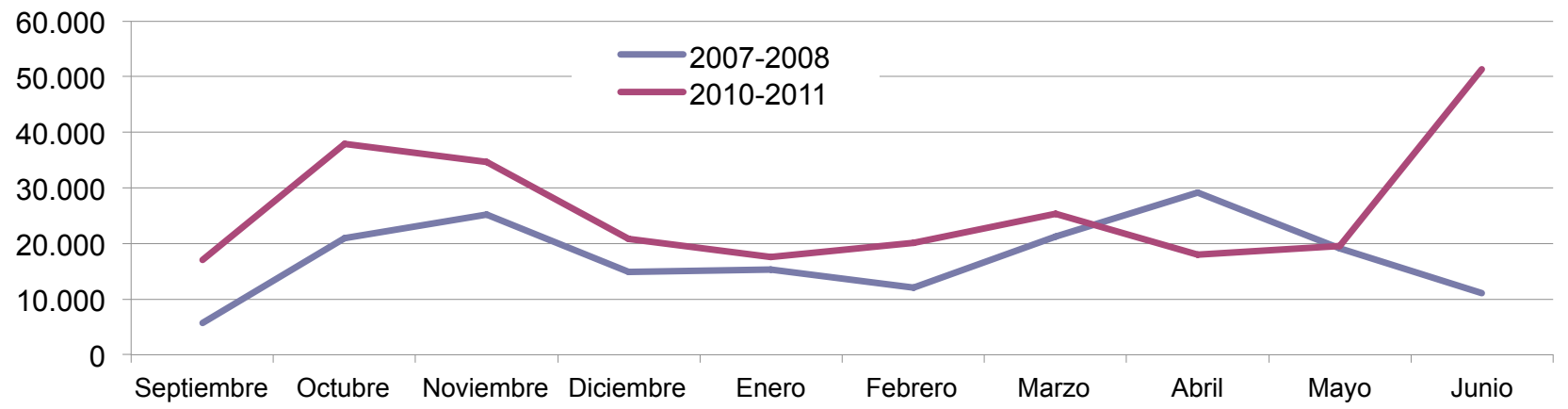

Figura 4. Comparación del número total de accesos

\section{Conclusiones}

Con la perspectiva de los tres cursos académicos y el curso 2011-12 iniciado se pueden apuntar algunas conclusiones sobre la experiencia de estos tres años de la asignatura de Técnicas de búsqueda y uso de la información y la colaboración entre el Departamento de Biblioteconomía y Documentación y el Servicio de Biblioteca de la Universidad Carlos III de Madrid.

La implementación de este modelo de asignatura transversal en todas las titulaciones de primer curso tiene dos grandes ventajas: desde el punto de vista del estudiante y de los profesores, se logra mejorar las competencias informacionales de los estudiantes y que ello redunde en mejores trabajos de curso y una mayor concienciación sobre el uso de las citas y la necesidad de evitar el plagio. Desde el punto de vista de la biblioteca, que se alcanza un impacto en prácticamente el $100 \%$ de los estudiantes, lo que redunda en una mayor utilización de los recursos de información a lo largo de la vida del estudiante y un mejor aprovechamiento de la inversión que hace la Universidad en suscribir y gestionar fuentes de información y bases de datos multidisciplinares y especializadas.

Pero el modelo también tiene algunos inconvenientes: el primero, la cantidad de recursos humanos necesarios para organizar e impartir clases en un solo cuatrimestre a casi 3000 alumnos por año, lo que requiere un buen número de profesores y grandes esfuerzos por parte del servicio de biblioteca. El segundo, que lograr la aplicación del modelo requiere un alto compromiso tanto por parte de las autoridades académicas como de los estudiantes y departamentos y servicios implicados.

La colaboración entre el Departamento de Biblioteconomía y Documentación y el Servicio de Biblioteca ha representado una oportunidad para reforzar la cooperación entre los docentes y los bibliotecarios. Para la Biblioteca, ha supuesto conocer mejor las necesidades de los profesores y los alumnos, reflexionar sobre la adecuación de los servicios y las colecciones a dichas necesidades, y mejorar la organización y los procesos internos de trabajo. Para los profesores, la colaboración supone una actualización continua y un conocimiento más profundo de los recursos que adquiere la Biblioteca; un acercamiento a las dificultades de búsqueda y resolución de problemas de información que plantean profesores y estudiantes; y el establecimiento de sinergias de cooperación entre los docentes y el personal de biblioteca.

La buena acogida generalizada de la asignatura por parte de los estudiantes y sus resultados académicos mayoritariamente positivos sugiere que los alumnos han comprendido la importancia de saber buscar y gestionar información no sólo pensando en sus estudios universitarios, sino también de cara al futuro ejercicio profesional.

En suma, la implantación de la asignatura de Técnicas de búsqueda y uso de la información con los nuevos planes de Grado de la Universidad Carlos III de Madrid es un paso decisivo hacia la integración de la alfabetización en información en el currículo de los estudiantes universitarios. Con la asignatura se contribuye a formar profesionales autónomos, reflexivos y críticos, uno de los objetivos prioritarios del Espacio Europeo de Educación Superior. Queda como reto lograr ampliar la formación avanzada de estas competencias en cursos de posgrado, más orientados hacia la investigación que hacia las habilidades básicas en la búsqueda y uso de la información.

\section{Referencias}

ACRL (2000). Normas sobre aptitudes para el acceso y uso de la información en la educación superior. // Boletín de la Asociación Andaluza de Bibliotecarios. 60 (julio- 
septiembre). http://www.aab.es/pdfs/baab60/60a6.pdf (26/10/2011)

Aguilera, Raúl; Hernández Pérez, Tony; Ramos, Mayte; Vianello, Marina (2010). Enseñando a buscar y a utilizar información estadística: una colaboración conjunta entre docentes y personal de la biblioteca. // World library and information congress: 76th IFLA general conference and assembly, 10-15 August, Gothenburg, Sweden. http://www.ifla.org/files/hq/papers/ifla76/86-aguileraes.pdf (26/10/2011).

Area, Manuel (2007). Adquisición de competencias en información: una materia necesaria en la formación universitaria: documento marco de REBIUN para la CRUE. REBIUN. http://www.rebiun.org/export/docReb/Adquisi ciondecompetencias.doc (26/10/2011).

Area, Manuel; Gros, Begoña; Marzal, Miguel Ángel (2008). Alfabetizaciones y tecnologías de la información y la comunicación. Madrid: Síntesis.

Bundy, Alan (ed.) (2004). Australian and New Zealand Information Literacy Framework: principles, standards and practice. Adelaide: ANZIIL. http://www.library.unisa. edu.au/learn/infolit/Infolit-2nd-edition.pdf (26/10/2011).

CIBER - Centre for Information Behaviour and the Evaluation of Research (2008). Information behaviour of the researcher of the future: A CIBER briefing paper. London: CIBER. http://www.jisc.ac.uk/media/documents/program mes/reppres/gg_final_keynote_11012008.pdf (29/10/ 2011).

Connaway, Lynn Silipigni; Dickey, Thimoty J (2010). The Digital Information Seeker: Report of the Findings from Selected OCLC, RIN, and JISC User Behaviour Projects. http://www.jisc.ac.uk/media/documents/publica tions/reports/2010/digitalinformationseekerreport.pdf (29/10/2011).

EEES: Espacio Europeo de Educación Superior. http://www.eees.es/es (26/10/2011).

ESPAÑA. Ministerio de Educación (2011). Estrategia Universidad 2015: contribución de las universidades al progreso socioeconómico español 2010-2015. Madrid: Ministerio de Educación. NIPO: 820-11-042-1. http://www.educacion.gob.es/eu2015.html (29/10/2011).

Hernández, Tony; Vianello, Marina; Ramos, Mayte; Aguilera, Raúl (2009). Técnicas de búsqueda y uso de la información: una nueva asignatura en los planes de Grado. // VII Jornadas CRAI: competencias informacionales e informáticas en el ámbito universitario (Madrid, 4 y 5 de junio). http://www.upm.es/eventos/JornadasCRAl/docs/ VIIJornadasCRAI-UC3M.pdf (26/10/2011).
Lau, Jesús (2006). Guidelines on Information Literacy for Lifelong Learning. // World Library and Information Congress: 72nd IFLA General Conference and Council. Libraries: Dynamic Engines for the Knowledge and Information Society. 20-24 August, Seoul, Korea. IFLA, 2006. http://archive.ifla.org/VII/s42/pub/IL-Guidelines2006.pdf (26/10/2011).

OCDE (2001). Understanding the digital divide. París: OCDE. http://www.oecd.org/dataoecd/38/57/1888451.pdf $(26 / 10 / 2011)$

OCDE (2005). La definición y selección de competencias clave DeSeCo. http://www.deseco.admin.ch/bfs/dese co/en/index/03/02.parsys.78532.downloadList.94248.Do wnloadFile.tmp/2005.dscexecutivesummary.sp.pdf (29/10/2011).

OCLC (2005). College Students' Perceptions of the Libraries and Information Resources: A Report to the OCLC Membership. Dublin, OH: OCLC, 2005. http://www. oclc.org/reports/pdfs/Percept_all.pdf (29/10/2011).

OCLC (2010). Perceptions of Libraries, 2010: Context and Community. Dublin, OH: OCLC, 2010. http://www.oclc. org/reports/2010perceptions/2010perceptions_all.pdf (29/10/2011).

Pinto, María; Sales, Dora; OSORIO, Pilar. Biblioteca universitaria, CRAI y alfabetización informacional. Gijón: Trea, 2008.

Pinto, M., Cordón, J. A.; Gómez Díaz, R (2010). Thirty years of information literacy (1977-2007): A terminological, conceptual and statistical analysis. // Journal of Librarianship and Information Science. 42:1, 3-19. DOI: 10.1177/0961000609345091

Shapiro, J. J.; Hughes, S. K (1996). Information literacy as a liberal art? // Educom review. 31, 31-35. http://www. si.umich.edu/ rfrost/courses/SI110/readings/InfoTrads/in fo_literacy.pdf (29/10/2011)

SCONUL (1999). The Seven Pillars of Information Literacy. http://www.sconul.ac.uk/groups/information_literacy/pap ers/seven_pillars.html (26/10/2011).

UNESCO (2011). Media and Information Literacy Curriculum for Teachers. Edited by Alton Grizzle and Carolyn Wilson. Paris: UNESCO, 2011. ISBN: 978-92-3-104198-3. http://unesdoc.unesco.org/images/0019/001929/192971 e.pdf (29/10/2011).

Verniers, E. P (2009). Media literacy in Europe Controversies, challenges and perspectives. Bruxelles: EuroMeduc. http://www.euromeduc.eu/IMG/pdf/Euromeduc ENG.pdf (29/10/2011) 
